\title{
A Case of VBA Application in Transaction Processing
}

\author{
Guoliang Dong ${ }^{\mathrm{a}}$, Guofang $\mathrm{Wu}^{\mathrm{b}}$, Chaozhou Chen ${ }^{\mathrm{c}}$ \\ Research Institute of Highway Ministry of Transport, Beijing, China \\ a'GL.DONG@rioh.cn, ${ }^{\mathrm{b}} \mathrm{GF} . W U @$ rioh.cn, ${ }^{\mathrm{C}} \mathrm{CZ} . C h e n @$ rioh.cn
}

Keywords: VBA, Transaction Processing, Excel, Word, Mapping Table, Efficiency

\begin{abstract}
Plenty of transactional work is continual, odd and time-consuming. Using the VBA technique can simplify the work procedure and liberate the workforce. A case of fetching required information from Word files and filling into the sheet of an Excel file is discussed. A general method of using embedded VBA codes and a mapping table is proved to be efficient, easy to implement and meet the demand.
\end{abstract}

\section{Introduction}

There is some transactional work which is no technical difficulty but time-consuming and odd in our daily work [1]. Using proper tools and methods, these affairs can be completed in a short while and the error can be decreasing to zero with a very little additional work [2,3]. A lot of successful cases using VBA to solve the odd and time-consuming routine affairs is reported [4,5,6]. Using embedded VBA program in Word or Excel documents [7,8], plenty of odd affairs work come to be easy which liberate the operators and improve the efficiency [9]. An association is newly built. The membership should be audit. A hundred of application lists are applied. Each applicant submits a form in a Word file. The information of the applicants should be summarized together as a new sheet. Using embedded VBA in an Excel file and programming a little additional code to open each Word file and fetch the information from the table and summarize as a new Excel file [10].

\section{Affair Description}

In order to be a membership of the association, the applicants are demanded to fill a form which includes personal information and other technique specialty. Each applicant should submit the information in a Word file. Hundreds of applicant's files have been received and should be summarized together.

\section{Transaction Processing with VBA}

Each item is saved in the table cell of a Word file. Locate the position of the required cell in the table. Establish a mapping table between the demanded information and the location in the Word-file's table. Dynamically modify the mapping table to get different cells' contents.

Personal Information. Each applicant fill the form which will be saved as a Word file. A part of applicant's information is shown in Table 1.

Mapping Table. In order to summarize the applicants' information, the Word files of the applicants should be traversal accessed. Fetch the required information from the file and fill into the sheet of the Excel file.

Establish a mapping table between the required information and the cell's location in the Word file. Modify the mapping table to get different information. An example of the mapping table is shown in Table 2.

VBA Implement. In an Excel file using the embedded VBA codes to implement the traversal access of the Word files, fetch the required information form the table of the Word files and fill the sheet. 
Table 1 Part of personal information

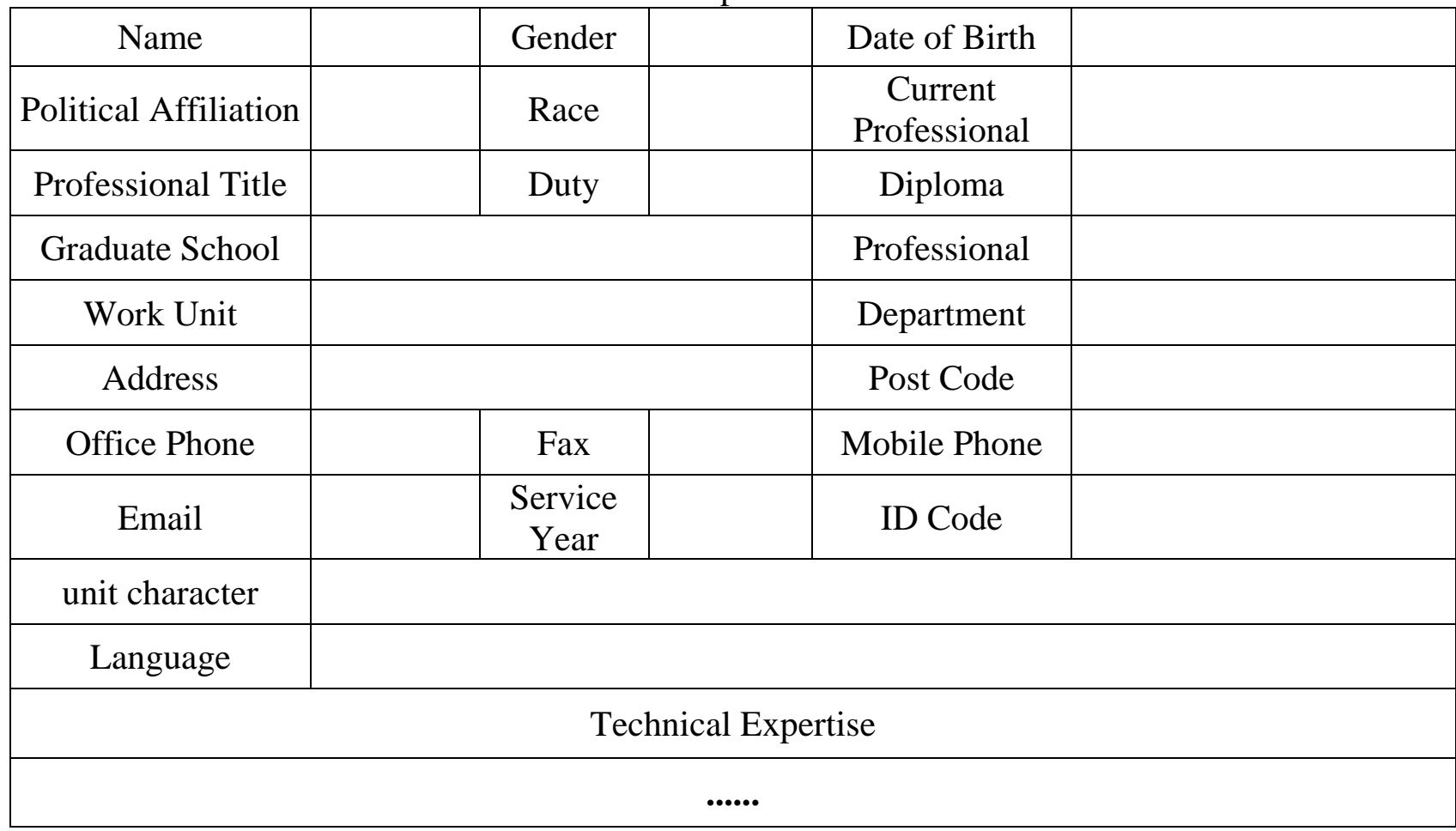

Table 2 an example of the mapping table

\begin{tabular}{|c|c|c|c|}
\hline No. & Item & Row & Column \\
\hline 1 & Name & 1 & 2 \\
\hline 2 & Gender & 2 & 4 \\
\hline 3 & Current Professional & 3 & 6 \\
\hline 4 & Duty & 2 & 4 \\
\hline 5 & Diploma & 4 & 2 \\
\hline 6 & Graduate School & 5 & 2 \\
\hline 7 & Work Unit & 6 & 2 \\
\hline 8 & Address & 6 & 4 \\
\hline 9 & Post Code & 7 & 4 \\
\hline 10 & Fax & 7 & 6 \\
\hline 11 & Mobile Phone & 8 & 2 \\
\hline 12 & Email & 12 & 1 \\
\hline 13 & Technical Expertise & 1 & 6 \\
\hline 14 & Date of Birth & 2 & 2 \\
\hline 15 & Political Affiliation & 2 & 4 \\
\hline 16 & Race & 4 & 4 \\
\hline 17 & Professional & 5 & 4 \\
\hline 18 & Department & 7 & 2 \\
\hline 19 & Office Phone & $\ldots \ldots$ \\
\hline$\ldots \ldots$ & $\ldots \ldots$ & $\ldots$ & $\ldots$ \\
\hline
\end{tabular}

Preparation. The preparation function includes getting the path of the Word files and the save-as Excel file, the save-as Excel name and the mapping table etc. Part programming codes are list as below.

Worksheets(mainSheet). Activate 


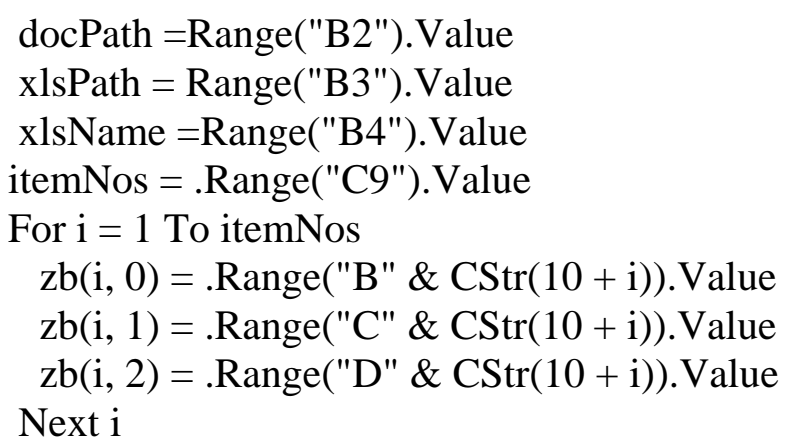

Traversal Accessing of the Word Files. Before opening the Word files it is necessary to search the path and get the file names of the Word files. Part programming codes are list as below.

Set Fso = CreateObject("scripting.filesystemobject")

For Each $\mathrm{f}$ In Fso.GetFolder(p).Files

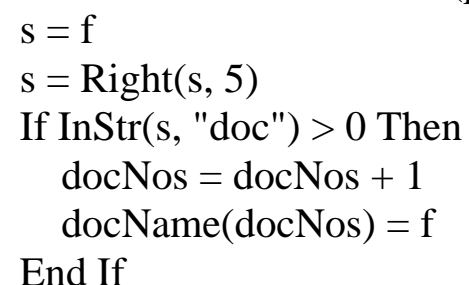

Next

Fetching the Required Information from Word Table. Open each Word file, get the required information from the file and fill the correspondent cell in the sheet based on the mapping table. Part programming codes are list as below.

Set wdApp = CreateObject("Word.Application")

wdApp.Visible $=$ True

With wdApp

For docFileNos $=1$ To docNos

.Documents.Open Filename:=docName(docFileNos), revert:=False

.ActiveDocument.SaveAs Filename:="t\$empp.doc "

With .ActiveDocument.tables(1)

For items $=1$ To itemNos

rowNoInWordTable $=\operatorname{Int}(\operatorname{Val}(z b($ items, 1$)))$

columnNoInWordTable $=\operatorname{Int}(\operatorname{Val}(z b($ items, 2$)))$

.Cell(rowNoInWordTable, columnNoInWordTable).Select itemStr(items) $=$ wdApp.Selection. Text

Next items

End With

.ActiveDocument.Close SaveChanges:=wdDoNotSaveChanges

With Workbooks(ThisXslFileName).Worksheets(summarySheet)

For $\mathrm{i}=1$ To itemNos

Next i

.Cells(docFileNos, 1). Value $=$ itemStr(i)

End With

Next docFileNos

End With

wdApp.Quit

Set wdApp $=$ Nothing

Sheets(summarySheet).Copy

ActiveWorkbook.SaveAsFilename:=summaryXlsFileFullName,

FileFormat:=xlOpenXMLWorkbook, CreateBackup:=False

ActiveWindow.Close

Workbooks(ThisXslFileName).Activate 
Worksheets(summarySheet).Activate

\section{Conclusion}

Summarizing personal information from lots of files is continual and time-consuming. Using a little additional VBA codes simplify the work procedure and liberate the workforce [11]. The mapping table provides an easy method to get different cells from the Word file. The method discussed here has met the demand and is proved to be efficient and easy to implement.

\section{References}

[1] Feng Min, Mao Shan-feng. Design of A Program for Vast Journals Metadata Automatic Extraction [J]. Chinese Journal of Scientific and Technical Periodicals, 2016 27(10)1081-1084.

[2] Liang A-quan,Liang Yue, Ma Bao-jun, Li Tai-yan, Gao Shuang-ling. Conversion of Earthquake Catalog Format by application of VBA Excel [J]. Plateau Earthquake Research, 2016 28(4)59-62.

[3] Jiang Yu-qi. A General Report Summarizing Program by VBA [J]. Computer Programming Skills \& Maintenance, 2017(2)48-49.

[4] Liu Sheng-da, Dong Ling. Using Excel VBA to Realize Statistical Analysis on the Quantitative Character of Maize [J]. Intelligent Computer and Applications, 2016 6(6)100-102.

[5] Yao Zhe. The Application of Excel VBA for Financial Works [J]. China Management Informationization , 2016 19(21)65-69.

[6] Chen Bao-ru, Wang Gui-lian, Song Zhang-di. The Application of Excel and VBA in Educational Administration System [J]. Home Drama, 2016(6)174-175.

[7] Zhang Liang. A Quick Document-Generation Tool Based on Excel VBA [J]. Computer Knowledge and Technology, 2016 12(32)264-265.

[8] Zhang Hong-fei. Automatic Generator of the Detector Report with VBA [J]. Fujian Computer, 2016(9)129-130.

[9] Guan Xiao-min, Yang Zhong-lu, Chen Hai-fen etc. Design and Application of Agronomic Traits of Regional Trial Data Entry System Based on Excel VBA [J]. Journal of Agriculture, 2016 6(8)54-58.

[10] Wang Shou-yin. Search Some Required Content in Word Files with VBA [J]. Computer Programming Skills \& Maintenance, 2016(16)10-11.

[11] Jiang Yu-qi. A Method of Batch Exporting Score Notes by VBA [J]. Computer Programming Skills \& Maintenance, 2016(6)38-39. 